\title{
A Tool for the Automatic Analysis of Single Events Effects on Electronic Circuits
}

\author{
Fernando García-Redondo, Marisa López-Vallejo, Pablo Royer, Javier Agustín
}

\begin{abstract}
Nowadays integrated circuit reliability is challenged by both variability and working conditions. Environmental radiation has become a major issue when ensuring the circuit correct behavior. The required radiation and later analysis performed to the circuit boards is both fund and time expensive. The lack of tools which support pre-manufacturing radiation hardness analysis hinders circuit designers tasks. This paper describes an extensively customizable simulation tool for the characterization of radiation effects on electronic systems. The proposed tool can produce an in depth analysis of a complete circuit in almost any kind of radiation environment in affordable computation times.
\end{abstract}

\section{INTRODUCTION}

Nowadays radiation tolerance of electronic circuits is becoming a hot topic for several reasons. First, there is a clear need of protecting electronic systems from radiation in specific environments: nuclear plants, particle accelerators and satellite or space missions. Second, with CMOS coming deep in the nanometer regime, the reliability of integrated circuits has become a major issue for circuit designers. It has been reported for SRAMs that the single-bit soft error rate decreases with scaling whereas the multi-bit single event rate (SER) increases dramatically [1].

Circuit designers lack of radiation analysis or simulation tools to check robustness of the radiation hardness techniques they implement. Circuits need to be fully implemented and go to expensive radiation tests in acceleration facilities, making the design cycle extremely long, expensive and unafordable for most companies. In this scenario, systematic analysis methods and tools that emulate the effects of radiation in electronic circuits are required. In this work we present a tool that satisfies these needs, providing a fast, reliable and customizable radiation analysis for any kind of circuit. The proposed tool carries out a complete analysis that allows the study of the damage caused by a single event transients (SET), the possible single event upsets (SEUs) generated and their propagation through the circuit paths. The tool has been conceived to provide two main targets:

- Conscientious analysis and search of the weakest circuit modules, allowing the designer to strengthen them before sending the manufactured circuit to a physical radiation test.

- Detection of the critical amount of charge that, injected at each node, produces a functional error. This functionality is especially interesting when designing circuits which can be exposed to different radiation environments.
To fulfill the above objectives the tool systematically analyzes the circuit structure at the transistor level (netlist), injects a simulated radiation at each susceptible circuit instance and easily determines the circuit critical module which is most sensitive to radiation.

Particle impact modeling, especially SETs generation and propagation through devices, has been studied in several publications [2]-[6]. However, the reliability-performance trade-off limits the scope of those techniques when applied to circuits bigger than a small quantity of transistors [7]. Other articles describe single event upsets (SEUs), generated by one or more SETs, as well as their propagation over HDL circuit models [8], [9]. Although the circuit range gets extended by this kind of analysis, their binary treatment leaves out deeper analysis such as how one or more SETs break into a SEU. This paper proposes a new radiation simulation tool to solve those needs. The simulation tool carries out a previous analysis of the circuit, detecting the nodes/instances susceptible of being catch up by a particle impact. Afterwards our tool systematically simulates radiation of the circuit finding the weakest modules and nodes. Not only does it find the most sensitive areas but it also determines the critical charge with which, at each impacted node/circuit instance, a SEU will be generated.

The paper is structured as follows. The main modules and functionalities of the tool are described in section II. Section III contains a detailed example of use, describing the radiation tool capabilities. Finally, some conclusions are drawn.

\section{TOOL DESCRIPTION}

This section describes the available radiation schemes, the main modules and functionalities of the proposed tool (shown in figure 1), and the radiation analysis key points (radiation error detection and granularity considerations).

\section{A. Radiation Schemes}

Our radiation tool considers two powerful radiation schemes which, following the configurability provided, model almost any kind of SET phenomena. The designer can choose one of the following approaches:

- Injection Scheme. The tool places a current source at each node susceptible of being radiated. The current source injects any waveform the user defines in order to accurately materialize the ion/wave impact. Most commonly used waveforms refer to the double exponential, PWD signals or simple pulses [4], [6], [8]. 


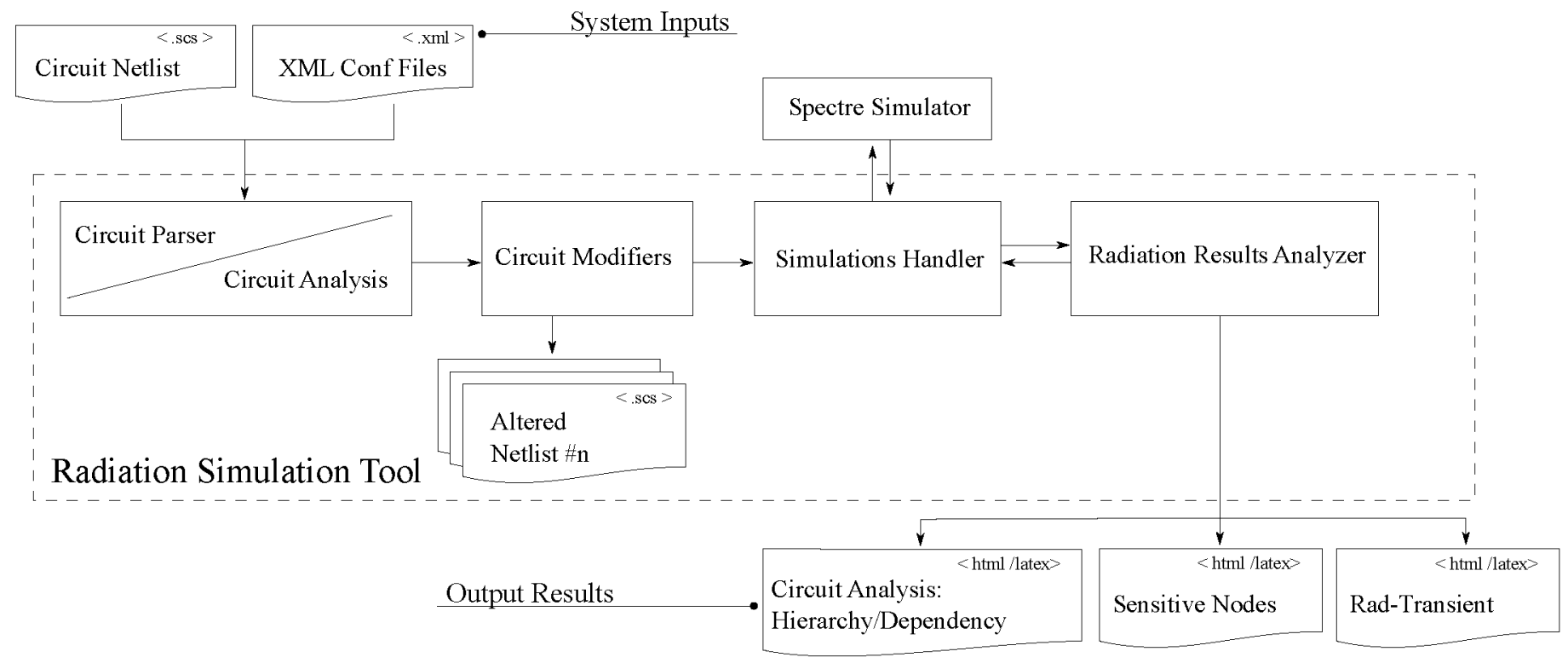

Fig. 1. Circuit Radiation Tool Flow Graph. The modular-system is represented along the required inputs and the generated outputs.

a) Bias Dependent

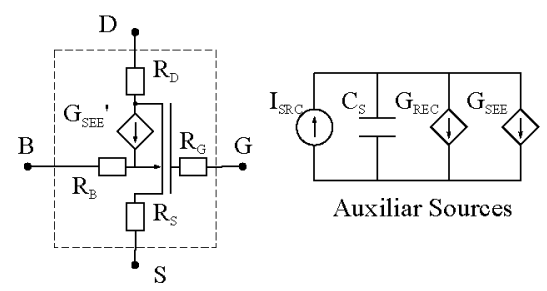

Fig. 2. Substitution scheme examples: a) Bias dependent radiation subcircuit scheme [5] and b) Switch controlled radiation scheme [3].

- Substitution Scheme. The tool replaces any instance susceptible of being radiated with the instance of a radiated subcircuit. This radiation scheme allows our radiation tool to use complex radiation sources. Highly dependent on the technology, the accurate results of these radiation schemes usually rely on a previous calibration. Figure 2 shows two examples of this kind of sources: Bias dependent radiation subcircuit scheme [5] and switch controlled radiation scheme [3]. Many other complex models can be included using this approach such as [2].

For convenience, whenever we talk about radiation within the tool this means an emulated radiation occurrence.

\section{B. Tool Description}

A key feature in our radiation tool is its configurability through $X M L$ configuration files. The user can customize the program with several technologies and simulation options. In addition, the radiation environment definition is highly moldable, allowing the full customization of radiation impacts modeling. Besides these features, several simulation granularity/refinement fields, as shown in table I, are available to the user, incrementing the amount and types of target circuits to be analyzed.
TABLE I. MAIN AVAILABLE CUSTOMIZATION FIELDS.

\begin{tabular}{|c|c|}
\hline Simulator & $\begin{array}{l}\text { System Calls and Arguments } \\
\text { Primitives Definitions } \\
\text { Control Statements Definitions } \\
\text { Netlist Keywords }\end{array}$ \\
\hline Technology & $\begin{array}{l}\text { Transistors and Models } \\
\text { Unalterable Nodes } \\
\text { Technology Scanned Folders } \\
\text { Technology Omitted Folders }\end{array}$ \\
\hline Experiment & $\begin{array}{l}\text { Report Configuration } \\
\text { Transient Configuration } \\
\text { Error definition/Observable Signals } \\
\text { Radiation Scheme Configuration: } \\
\text { - Radiation Sources } \\
\text { - Radiation Parameters } \\
\text { - Injectable/Unalterable Statements and Nodes }\end{array}$ \\
\hline
\end{tabular}

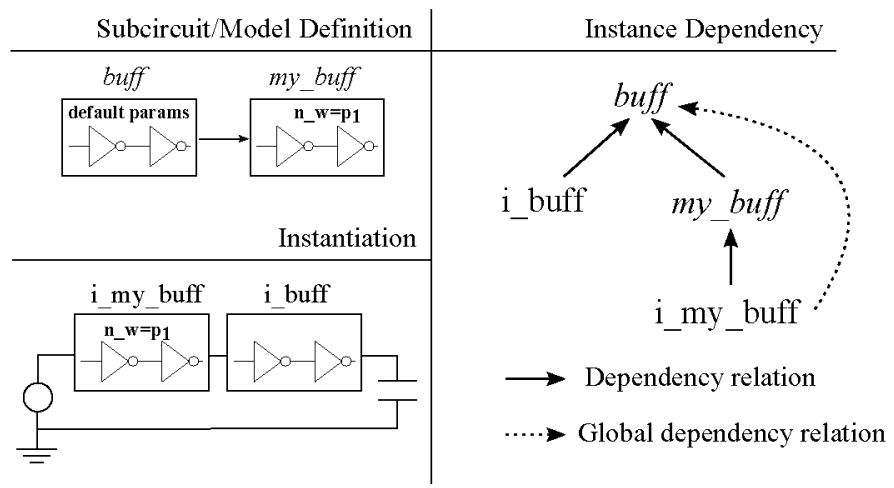

Fig, 3. Dependency Analysis Example.

Let's describe the proposed radiation tool structure, represented in figure 1 . It is composed of four main modules:

1) Circuit Analysis: This module is responsible of accurately parsing the input, Spectre netlists, to an assemble set of circuit instances structured as relational trees. Afterwards inheritance analysis takes place, organizing the different circuit elements, analysis and control statements and building relational tree structures. 


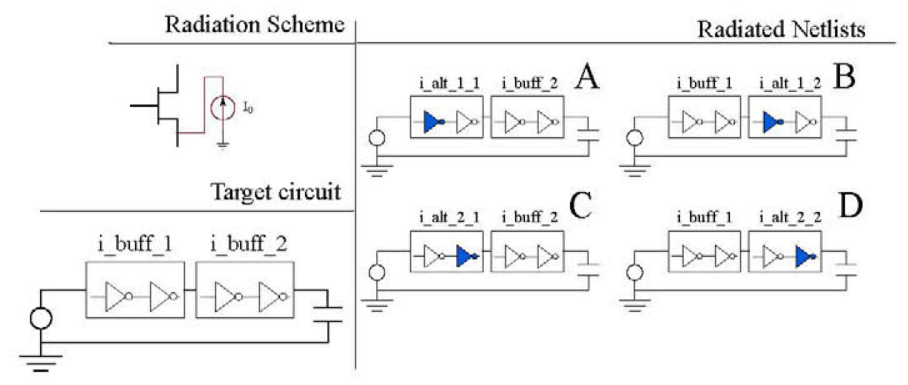

Fig. 4. Modifiers Example.

Finally, this module performs an instance dependency analysis which is required by the radiation algorithm. The dependency analysis allows, as shown in figure 3 , to directly access the primitives, (analog) models, subcircuits etc. bypassing non-desired statements and providing a useful search engine.

2) Circuit Modifiers: This module radiates the golden circuit at each element (node or instance) susceptible of being radiated by introducing a modifier which emulates radiation. The radiation algorithm recursively searches among the circuit elements independently of the number of levels the circuit has. Each radiation occurrence gets translated into a new altered netlist which is independently simulated and analyzed. The tool makes use of the relational data structures to find those perceptible nodes, and to propagate the radiation occurrence to dependent instances on upper levels of the circuit.

Figure 4 illustrates the way the modifiers work. The golden circuit contains two buffer subcircuit instances: i_buff_1 and i_buff_2. Each buffer instance is composed of a chain of two inverters. The selected radiation scheme is node injection. For simplicity, we will consider both $v_{-} i n, v d d$ and gnd as not-susceptible of being radiated nodes.

The alteration process is as follows:

1) The tool finds the first susceptible node of being modified by the injected radiation: the output of the first inverter in the buffer i_buff_1. The modifiers create an altered buffer subcircuit in which the first inverter is radiated alt_1. Right after this the modifiers substitute the original instance, i_buff_1, with the radiated one, i_alt_1_1 and export the radiated netlist $(A)$.

2) Before injecting any other circuit node, the modifiers propagate the subcircuit buffer alteration through the circuit: the buffer i_buff_2 gets substituted by i_alt_1_2 and a new radiated netlist is generated (B).

3) No more buffer instances are found, and therefore the modifiers radiate the next susceptible node, the second inverter of the first buffer i_buff_1. A new altered buffer subcircuit is created, alt_2, and the instance i_buff_1 is replaced by a radiated one i_alt_2_1 giving another radiated netlist $(C)$.

4) The modifiers propagate the last subcircuit buffer alteration: the second instance buffer i_buff_2 is substituted by a radiated instance i_alt_2_2, generating the last netlist $(D)$.

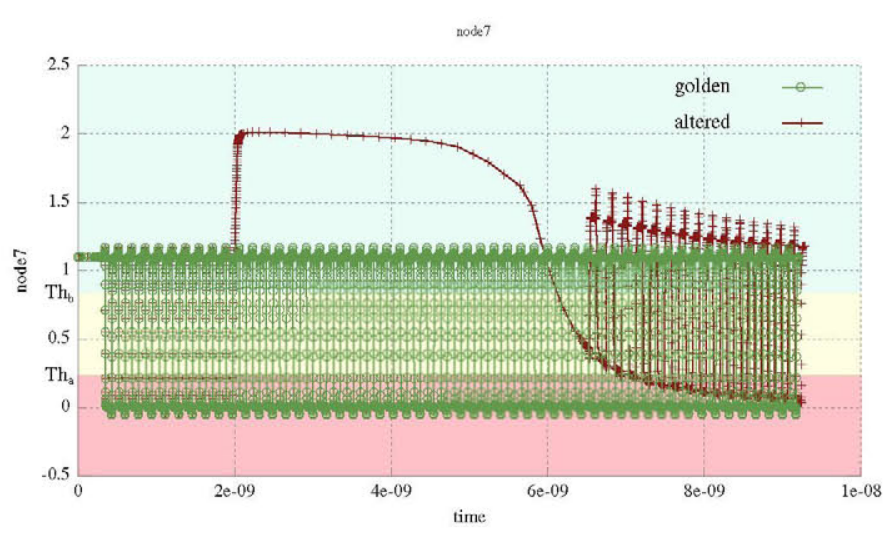

Fig. 5. Example of user defined signal regions in a $1.1 \mathrm{~V}$ fed ring oscillator circuit: a) Blue region for $v d d$ values, b) Red region for gnd values, c) Yellow region for transitions.

At this point no more nodes are susceptible of being radiated, and four different altered netlists emulating a SET occurrence at different places are ready to be simulated.

3) Simulations Handler: This module independently manages the simulation of each altered circuit generated by the previous module. It communicates with Spectre circuit simulator, concurrently handling every scenario simulation and redirecting the outputs to the Results Analysis module. In case that any simulation unexpectedly ends or an error occurs, the Simulations Handler reports the incident to the user. Otherwise, the transient results are ready to be analyzed.

4) Radiation Results Analysis: This module interpolates the results from the simulated scenarios and compares them with the golden circuit response. The error detection algorithm, explained in detail in section II-C, decides whether or not the radiation occurrence has become a functional error.

With the aim of finding the critical amount of charge with which the radiation occurrence at a given node produces a circuital error, this module interacts with the Simulations Handler; depending on the existence of an error, it will increase/decrease the total amount of charge in the next simulation until it finds, with a predefined accuracy, the critical value that generates that error.

At this point, the tool analyzes each node susceptible of being radiated, and determines which of them, and with which critical charge, are sensitive to radiation. Right after this, the tool automatically generates the documentation related to the radiation capabilities.

\section{Radiation Error Detection}

Independently on its size, each circuit module should handle a different definition of radiation error relying on the function it develops. For example, it is reasonable that for a memory unit the radiation error gets defined by a bit flip. On the other hand, on a given analog processing path the radiation error could mean a small voltage difference with a duration long enough to be propagated to the next unit.

The proposed radiation tool gives the user the required weapons to decide which signals should be defined as observables. A functional error is defined by the comparison of 
those observable signals within a radiated circuit against the pertinent ones in the golden (non-radiated) circuit. The field Analyzed Magnitudes/Signals in table I contains several user defined customization options to set up this comparison:

- Set of observable signals.

- Thresholds and absolute error margins.

- The minimum length of the event to be considered as an error (time span) is also configurable. Punctual errors can also be managed.

Therefore, the tool proceeds to independently compare each observable signal with the same one in the golden scenario. If the difference between them exceeds a predefined value, whether it is punctual or maintained in time, a radiation error is considered. With this configurability, the user can define, for instance, when a SET becomes a SEU.

To improve the accuracy of the analysis the tool allows the consideration of up to three different sets of comparison parameters depending on the value of the analyzed magnitude. Additionally, the user can independently tune the values of the different customizable fields for each region. Figure 5 shows an example with three regions shown in colors while evaluating the voltage at the same node of a ring oscillator. In this scenario the first region, gnd values in red, has an absolute error value of $\operatorname{err}_{a}$ and considers errors with lengths greater than $t_{a}$. The second region, between the thresholds $T h_{a}$ and $T h_{b}$ (in yellow) has a different absolute error value, $e r r_{b}$, and considers punctual errors. The last region, $v d d$ values in blue, has an absolute error value of $e r r_{c}$ and considers errors with lengths greater than $t_{c}$. Under this configuration, a radiation error is found at $2 n s$ simulation time.

\section{Granularity}

A key point during any radiation simulation is the computation time, which dramatically increases when dealing with large circuits. Despite the proposed schematic simulation takes advantage of simpler models, especially when compared with TCAD-like simulations, the computation time remains as a key factor. We propose the use of a granularity strategy, analyzing several modules independently and extrapolating the radiationhardness properties to upper levels. Figure 6 represents an example of this strategy. First of all the user simulates and extracts the radiation properties of the individual modules, in this case, the two different buffers buff and my_buff with critical charges $Q_{1}$ and $Q_{2}$ respectively. Now, the user extrapolates those properties to the whole circuit. The circuit critical amount of charge will be $Q_{\text {buff_pair }}=\min \left(Q_{1}, Q_{2}\right)$.

In the second stage, making use of the customization capabilities, the user analyzes the remaining modules of the circuit omitting the pair of buffer instances saving computational resources. If instead of only having a pair of buffers we had a larger number, the computation time saved using this strategy would be determinant, allowing the simulation of larger circuits.

However, when considering not only combinational modules, we need to take into account the delays induced between modules. The signals registration will be delayed by the previous modules propagation time. Therefore, the SEU generation

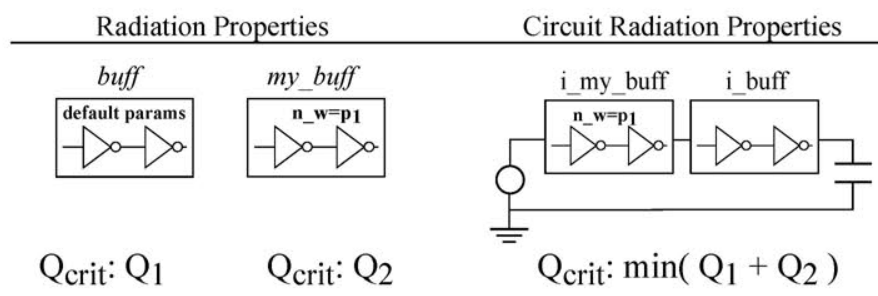

Fig. 6. Granularity strategy example.

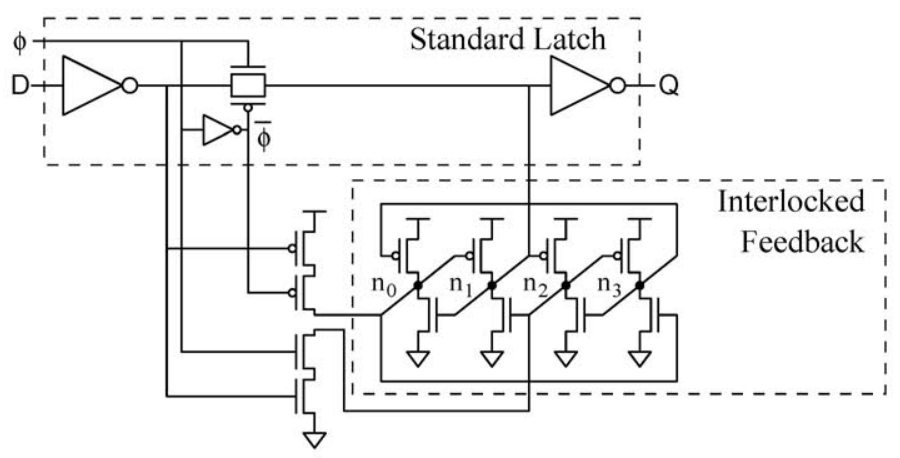

Fig. 7. DICE flip-flop schematic presented in [10].

will depend on the time the SET occurs, and so the value $Q_{\text {crit }}$ will depend on time becoming the function $Q_{\text {crit }}(t)$. Section III deals with this issue.

\section{EXAMPLE OF USE}

This section contains a guided example which shows the proposed tool capabilities. We have chosen two different flipflop circuits to analyze their hardness against radiation. Relying on the radiation tool, we will determine the radiation hardness of the two of them. The first circuit is a twelve standard flip-flop, built on $40 \mathrm{~nm}$ technology. The second circuit is a hardened flip-flop with a dual interlocked cell (DICE) structure [10]. The hardening principle is described in figure 7 , where the latch is improved by using the dual interlocked feedback circuitry underneath. Clock edges are always subject to error when dealing with timed modules. The DICE flip-flop should be more robust in non-clock transition instants, and we are make use of our tool to measure that improvement.

The first step is the experiment configuration:

- Technology: 40nm TSMC.

- Definition of the module timing: $10 \mathrm{~ns}$ clock period.

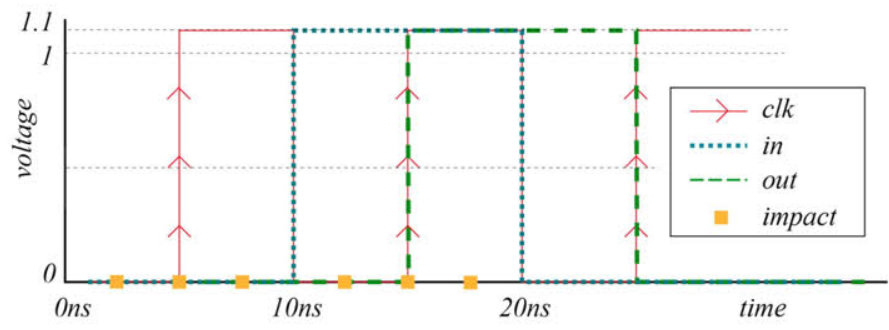

Fig. 8. Radiation impacts in the analyzed registers. 
TABLE II. Standard Register. CRITICAL CHARge AT EACH NODE VARYING THE PARTICLE TIME OF IMPACT, $\star$ : NOT AFFECTED BY THE MAXIMUM CHARGE $(1 E-11 C)$. $A B M C$ : AFFECTED BY THE MINIMUM CHARGE $(1 E-14 C)$

\begin{tabular}{|r|r|r|r|r|r|r|}
\hline$t_{i}(\boldsymbol{n s})$ & Node 0 & Node 1 & Node 2 & Node 3 & Node 4 & Node 5 \\
\hline 2.5 & $3.05 \mathrm{E}-012$ & $\star$ & $\star$ & $\star$ & $3.90 \mathrm{E}-13$ & $9.69 \mathrm{E}-012$ \\
5 & ABMC & $\star$ & $\star$ & $\star$ & $2.04 \mathrm{E}-14$ & $1.51 \mathrm{E}-13$ \\
7.5 & ABMC & $\star$ & $\star$ & $\star$ & $1.67 \mathrm{E}-14$ & $\star$ \\
12.5 & $\star$ & ABMC & $\star$ & $3.8 \mathrm{E}-14$ & $1.38 \mathrm{E}-12$ & $1.43 \mathrm{E}-14$ \\
15 & $\star$ & ABMC & $\star$ & $4.54 \mathrm{E}-14$ & $8.44 \mathrm{E}-12$ & $\star$ \\
17.5 & $\star$ & $\star$ & $\star$ & $\star$ & $9.06 \mathrm{E}-12$ & $\star$ \\
\hline$t_{i}(\boldsymbol{n s})$ & Node 6 & Node 7 & Node 8 & Node 9 & Node 10 & Node 11 \\
\hline 2.5 & $\star$ & $1.91 \mathrm{E}-14$ & $\star$ & $1.55 \mathrm{E}-14$ & $1.55 \mathrm{E}-14$ & $\star$ \\
5 & $\star$ & $\star$ & $\star$ & $\mathrm{ABMC}$ & $1.55 \mathrm{E}-14$ & $\star$ \\
7.5 & $\star$ & $\star$ & $\star$ & $\mathrm{ABMC}$ & $3.56 \mathrm{E}-14$ & $\star$ \\
12.5 & $1.30 \mathrm{E}-12$ & $6.12 \mathrm{E}-14$ & $\star$ & $\star$ & $\star$ & $\mathrm{ABMC}$ \\
15 & $1.55 \mathrm{E}-14$ & $\star$ & $\star$ & $\star$ & $\star$ & $\mathrm{ABMC}$ \\
17.5 & $3.56 \mathrm{E}-14$ & $\star$ & $\star$ & $\star$ & $\star$ & $\star$ \\
\hline
\end{tabular}

- Particle impact modeling: We will use the double exponential injection scheme [8] in which the particle impact generates a current at the impacted node with the form

$$
\begin{aligned}
i_{i n j} & =I_{0} *\left(e^{-\left(t-t_{i}\right) / \tau_{2}}-e^{-\left(t-t_{i}\right) / \tau_{1}}\right) \\
I_{0} & =Q_{\text {coll }} /\left(\tau_{2}-\tau_{1}\right)
\end{aligned}
$$

Parameters $\tau_{1}$ and $\tau_{2}$ depend on the technology. During our experiment we will consider the values $\tau_{1}=1 E-11(s)$ and $\tau_{2}=2 E-10(s) . Q_{\text {coll }}$ refers to the collected charge at the impacted node. The last parameter, $t_{i}$, is the particle impact instant.

- Maximum and minimum charge range: In our experiment, this parameter will vary between $1 E-14 C$ and $1 E-11 C$.

- Definition of the particle impact instants $t_{i}$ : between $2.5 \mathrm{~ns}$ and $17.5 \mathrm{~ns}$ avoiding the situations when both clock and input have transitions, as shown in figure 8 .

- Observable signals: The chosen observable signal is the output of each flip-flop.

- Definition of our circuit radiation error. We will consider a radiation error as the occurrence of a nondesired bit flip due a particle impact. This bit change should at least last $1 \mathrm{~ns}$ to be considered a SEU.

- Definition of injectable circuit components and nodes. Each component is susceptible of being radiated. Each node but the output, our observable signal, is susceptible of being impacted by a particle.

With the above configuration we proceed to simulate the experiment. After the radiation analysis, the results extracted from the report confirm the radiation robustness of the DICE scheme facing the standard flip-flop shown in [10]. Tables II and III summarize the results ordered by the circuit modules internal nodes. From its thorough analysis we extract the data summary shown in table IV which supports the DICE radiation resistance regarding the standard register: DICE structures, even having more nodes perceptible of being radiated, present a better radiation hardness behavior.

Moreover, we also deduce the worst impact time $t_{i}$ at which our module is more sensitive. As expected, clock rising edges are the most sensitive instants ( $5 \mathrm{~ns}, 15 \mathrm{~ns})$.
TABLE III. DICE REgISTER. CRITICAL CHARGE AT EACH NODE VARYING THE PARTICLE TIME OF IMPACT, $\star$ : NOT AFFECTED BY THE MAXIMUM CHARGE $(1 E-11 C)$. ABMC: AFFECTED B Y THE MINIMUM CHARGE $(1 E-14 C)$

\begin{tabular}{|r|r|r|r|r|r|r|}
\hline$t_{i}(\boldsymbol{n s})$ & Node 0 & Node 1 & Node 2 & Node 3 & Node 4 & Node 5 \\
\hline 2.5 & $\star$ & $\star$ & $\star$ & $\star$ & $\star$ & $\star$ \\
5 & $\star$ & $\star$ & $\star$ & $\star$ & $\star$ & $\star$ \\
7.5 & $\star$ & $\star$ & $\star$ & $\star$ & $\star$ & $\star$ \\
12.5 & $1.03 \mathrm{E}-13$ & $\star$ & $\star$ & $\star$ & $\star$ & $1.91 \mathrm{E}-14$ \\
15 & $2.27 \mathrm{E}-12$ & $\star$ & $\star$ & $\star$ & $\star$ & $2.04 \mathrm{E}-14$ \\
17.5 & $2.27 \mathrm{E}-12$ & $\star$ & $\star$ & $\star$ & $\star$ & $\star$ \\
\hline$t_{i}(\boldsymbol{n} \boldsymbol{s})$ & Node 6 & Node 7 & Node 8 & Node 9 & Node 10 & Node 11 \\
\hline 2.5 & $\star$ & $\star$ & $\star$ & $\star$ & $\star$ & $\star$ \\
5 & $\star$ & $\star$ & $\star$ & $\star$ & $\star$ & $\star$ \\
7.5 & $\star$ & $\star$ & $\star$ & $\star$ & $\star$ & $\star$ \\
12.5 & $\star$ & $\star$ & $\star$ & $1.3 \mathrm{E}-12$ & $\star$ & $\star$ \\
15 & $\star$ & $\star$ & $\star$ & $1.22 \mathrm{E}-12$ & $\star$ & $\star$ \\
17.5 & $\star$ & $\star$ & $\star$ & $1.3 \mathrm{E}-12$ & $\star$ & $\star$ \\
\hline$t_{i}(\boldsymbol{n} \mathbf{s})$ & Node 12 & Node 13 & Node 14 & Node 15 & Node 16 & Node 17 \\
\hline 2.5 & $\star$ & $4.98 \mathrm{E}-13$ & $6.15 \mathrm{E}-13$ & $\star$ & $\star$ & $\star$ \\
5 & $\star$ & $4.54 \mathrm{E}-14$ & $2.4 \mathrm{E}-14$ & $\star$ & $\star$ & $\star$ \\
7.5 & $\star$ & $1.53 \mathrm{E}-12$ & $\star$ & $\star$ & $\star$ & $\star$ \\
12.5 & $\star$ & $\star$ & $\star$ & $\star$ & $\star$ & $\star$ \\
15 & $\star$ & $\star$ & $\star$ & ABMC & $\star$ & ABMC \\
17.5 & $\star$ & $\star$ & $\star$ & $\star$ & $\star$ & $1.03 \mathrm{E}-13$ \\
\hline
\end{tabular}

TABLE IV, CRITICAL CHARGE AT EACH NODE, NUMBER OF SENSITIVE NODES AND RATIO BETWEEN SENSITE NODES AND NODES SUSCEPTIBLE OF BEING RADIATED FOR EACH REGISTER SCHEME.

Standard
\begin{tabular}{|r|r|r|r|}
\hline$t_{\boldsymbol{i}}(\boldsymbol{n} \boldsymbol{s})$ & \# Sensitive Nodes & Ratio & Min $Q_{c}$ \\
\hline 2.5 & 6 & 0.5 & $1.55 \mathrm{E}-14$ \\
\hline 5 & 5 & 0.42 & $\mathrm{ABMC}$ \\
\hline 7.5 & 4 & 0.33 & $\mathrm{ABMC}$ \\
\hline 12.5 & 7 & 0.85 & $\mathrm{ABMC}$ \\
\hline 15 & 5 & 0.42 & $\mathrm{ABMC}$ \\
\hline 17.5 & 2 & 0.17 & $3.56 \mathrm{E}-14$ \\
\hline
\end{tabular}

DICE
\begin{tabular}{|r|r|r|r|}
\hline$t_{i}(\boldsymbol{n s})$ & \# Sensitive Nodes & Ratio & Min $Q_{c}$ \\
\hline 2.5 & 2 & 0.11 & $4.98 \mathrm{E}-13$ \\
\hline 5 & 2 & 0.11 & $2.4 \mathrm{E}-14$ \\
\hline 7.5 & 1 & 0.05 & $1.53 \mathrm{E}-12$ \\
\hline 12.5 & 3 & 0.17 & $1.91 \mathrm{E}-14$ \\
\hline 15 & 5 & 0.28 & ABMC \\
\hline 17.5 & 3 & 0.17 & $1.03 \mathrm{E}-13$ \\
\hline
\end{tabular}

\section{Sequential Circuits Granularity Considerations}

When dealing with granularity capabilities, we introduced in section II-D some considerations regarding sequential circuits.

Let's consider a new scenario with a chain of five DICE registers which maintains the same timing as the example before. In case we want to extrapolate the radiation characteristics of the single DICE analysis, we should not consider the worst cases of the isolated register alone but as an ensemble of timed modules. Otherwise, two different consequences can appear:

1) If the SEU at the output of the first register does not meet the rising edge of the clock signal, it is not captured by the second register, shown in figure 9.

2) If the SET inside the first register does not produce a SEU (it is not long enough), but it matches the rising edge of the clock signal, a SEU might occur in the second register, as shown in figure 10.

Even if the probability of impact in a specific node at the worst time could be really small, regarding sequential circuits we should consider the critical amount of collected charge as a time dependent function $Q_{c r i t}(t)$. For a simple chain of two 


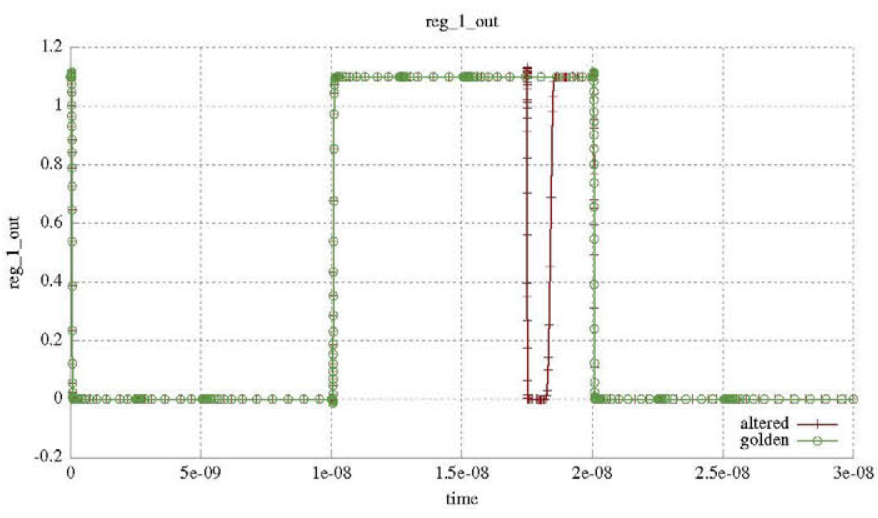

Fig. 9. The SEU generated in the first register will not be propagated to the second one. The SEU length is not long enough to be captured in the next clock edge (20ns).

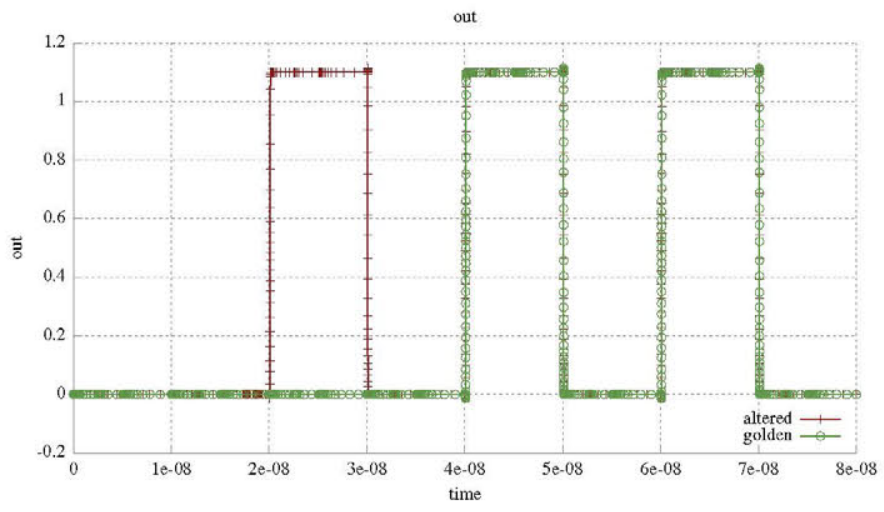

Fig. 10. The SEU generated in the first register at time $20 \mathrm{~ns}$ has been propagated till the output of our registers chain.

registers, being $Q_{r e g}(t)$ each register critical charge, and $d_{r e g}$ the delay between its input and output, we would consider $Q_{\text {pair }}$, the critical charge of the module, as

$$
Q_{\text {pair }}(t)=f_{\text {min }}\left(Q_{\text {reg }}(t), Q_{r e g}\left(t-d_{r e g}\right)\right) \text {. }
$$

However, the complexity of equation 3 grows depending on the number of register stages, complicating the calculus of the amount of charge that would destabilize our circuit behavior. Once again our radiation tool proves useful systematically solving the problem: The design space exploration of the five stage chain, involving fifty impacted nodes analyzed at six different instants was swiftly computed. It took over five minutes of a virtualized bicore Intel i7 with 8GB RAM to generate the radiation results shown in table V. As was predictable, some SEUs were corrected by posterior stages (2.5ns impact time). In other cases some SET propagations ended up becoming a SEU which previously was not present.

\section{TABLE V. DICE Chain Summary.}

\begin{tabular}{|r|r|r|}
\hline$t_{i}(\boldsymbol{n s})$ & \# Sensitive nodes & Min $Q_{c}$ \\
\hline 2.5 & 0 & $\star$ \\
5 & 11 & $2.16 \mathrm{E}-14$ \\
7.5 & 7 & $2.03 \mathrm{E}-14$ \\
12.5 & 2 & $1.91 \mathrm{E}-14$ \\
15 & 10 & $\mathrm{ABMC}$ \\
17.5 & 5 & $4.78 \mathrm{E}-14$ \\
\hline
\end{tabular}

\section{CONCLUSIONS}

Radiation has become a serious problem in current circuit reliability. Circuit designers require tools for the validation of the designs before being manufactured, saving costs and time. In this paper we have presented a useful radiation simulation tool which solves those circuit designers needs. A fast, powerful and reliable application framework that allows the user to test the circuit radiation hardness and allows finding the weakest modules in the circuit. The proposed radiation tool is fully customizable, allowing the user to model a wide range of radiation environments. Finally a representative example validated the tool and has illustrated its capabilities.

\section{ACKNOWLEDGEMENTS}

This work was funded by CICYT project TOLERA TEC2012-31292 of the Spanish Ministry of Economy and Competitiveness.

\section{REFERENCES}

[1] N. Seifert, P. Slankard, M. Kirsch, B. Narasimham, V. Zia, C. Brookreson, A. Vo, S. Mitra, B. Gill, and J. Maiz, "Radiation-induced soft error rates of advanced cmos bulk devices," in Reliability Physics Symposium Proceedings, 2006. 44th Annual., IEEE International. IEEE, 2006, pp. 217-225.

[2] T. Gadlage, M.J. and Schrimpf, R.D. and Benedetto, J.M. and Eaton, P.H. and Turflinger, "SEU and SET Modeling and Mitigation in Deep Submicron Technologies," in Reliab. Phys. Symp. Proceedings, 2004. 42nd Annu. 2004 IEEE Int., 2004.

[3] A. Makihara, T. Ebihara, T. Yokose, Y. Tsuchiya, Y. Amano, H. Shindou, R. Imagawa, Y. Takahashi, and S. Kuboyama, "New SET Characterization Technique Using SPICE for Fully Depleted CMOS/SOI Digital Circuitry," IEEE Trans. Nucl. Sci., vol. 55, no. 6, pp. 29212927, Dec. 2008.

[4] A. M. Francis, D. Dimitrov, J. Kauppila, A. Sternberg, M. Alles, J. Holmes, and H. A. Mantooth, "Significance of Strike Model in Circuit-Level Prediction of Charge Sharing Upsets," IEEE Trans. Nucl. Sci., vol. 56, no. 6, pp. 3109-3114, Dec. 2009.

[5] J. S. Kauppila, A. L. Sternberg, M. L. Alles, A. M. Francis, J. Holmes, O. A. Amusan, and L. W. Massengill, "A Bias-Dependent Single-Event Compact Model Implemented Into BSIM4 and a $90 \mathrm{~nm}$ CMOS Process Design Kit," IEEE Trans. Nucl. Sci., vol. 56, no. 6, pp. 3152-3157, Dec. 2009.

[6] K. Castellani-Coulie, G. Toure, J.-M. Portal, O. Ginez, H. Aziza, and A. Lesea, "Circuit Effect on Collection Mechanisms Involved in Single Event Phenomena: Application to the Response of a NMOS Transistor in a $90 \mathrm{~nm}$ SRAM Cell," IEEE Trans. Nucl. Sci., vol. 58, no. 3, pp. 870-876, Jun. 2011.

[7] W. G. Bennett, R. D. Schrimpf, N. C. Hooten, R. A. Reed, J. S. Kauppila, R. A. Weller, K. M. Warren, and M. H. Mendenhall, "Efficient Method for Estimating the Characteristics of Radiation-Induced Current Transients," IEEE Trans. Nucl. Sci., vol. 59, no. 6, pp. 2704-2709, Dec. 2012.

[8] V. Ferlet-Cavrois, L. W. Massengill, and P. Gouker, "Single Event Transients in Digital CMOS-A Review," IEEE Trans. Nucl. Sci., vol. 60, no. 3, pp. 1767-1790, Jun. 2013.

[9] H. M. Quinn, D. A. Black, W. H. Robinson, and S. P. Buchner, "Fault Simulation and Emulation Tools to Augment Radiation-Hardness Assurance Testing," IEEE Trans. Nucl. Sci., vol. 60, no. 3, pp. 2119 2142, Jun. 2013

[10] B. Stackhouse, S. Bhimji, C. Bostak, D. Bradley, B. Cherkauer, J. Desai, E. Francom, M. Gowan, P. Gronowski, D. Krueger, C. Morganti, and S. Troyer, "A $65 \mathrm{~nm}$ 2-billion transistor quad-core itanium processor," IEEE J. Solid-State Circuits, vol. 44, no. 1, pp. 18-31, 2009. 